\title{
Light echoes of SNe in the LMC
}

\author{
Armin Rest ${ }^{1}$, Nicholas B. Suntzeff ${ }^{2}$, R. Chris Smith ${ }^{1}$, Knut Olsen ${ }^{1}$, \\ Alfredo Zenteno ${ }^{1}$, Guillermo J. Damke ${ }^{1}$, Jose L. Prieto ${ }^{1}$, Christopher \\ Stubbs $^{3}$, Arti Garg ${ }^{3}$, Peter Challis ${ }^{2}$, Andrew C. Becker ${ }^{4}$, Gajus A. \\ Miknaitis ${ }^{4}$, Antonino Miceli ${ }^{4}$, Douglss L. Welch ${ }^{5}$, Alejandro \\ Clocchiatti $^{6}$, Dante Minniti ${ }^{6}$, Lorenzo Morelli ${ }^{6}$, Kem H. Cook $^{7}$, \\ Sergei Nikolaev ${ }^{7}$, Mark E. Huber ${ }^{7}$, and Andrew Newman ${ }^{8}$ \\ ${ }^{1}$ National Optical Astronomy Observatory (NOAO)/Cerro Tololo Inter-American Observatory \\ (CTIO), La Serena, Chile \\ email: arest@ctio.noao.edu \\ ${ }^{2}$ Department of Physics \& Astronomy, Texas A \& M University, \\ 4242 TAMU, College Station, TX 77843 USA

\footnotetext{
${ }^{3}$ Physics Department, Harvard University, 17 Oxford Street, Cambridge, MA 02138, USA

${ }^{4}$ Department of Astronomy, University of Washington, Box 351580, Seattle, WA 98195, USA

${ }^{5}$ Dept. of Physics and Astronomy, McMaster University, Hamilton, Ontario, L8S 4M1, Canada

${ }^{6}$ Dept. of Astronomy, Pontificia Universidad Católica de Chile, Casilla 306, Santiago 22, Chile

${ }^{7}$ Lawrence Livermore National Laboratory, 7000 East Ave., Livermore, CA 94550, USA

${ }^{8}$ Department of Physics, Washington University, Saint Louis, MO 63130, USA
}

\begin{abstract}
The SuperMACHO project has discovered light echoes from 3 ancient SNe in the LMC. These SNRs are three of the six youngest in the LMC, and are classified as likely SN Ia based on X-ray data.
\end{abstract}

Keywords. stars: supernovae: general, ISM: supernova remnants, ISM: reflection nebulae

In recent years, light echoes have been discovered around some nearby extragalactic supernovae well after the explosion, most notably the light echoes from SN 1987A (Crotts 1988). However, to date no light echoes of historical SNe of Galactic or extragalactic origin have been discovered. In the SuperMACHO project, we have imaged the bar of the LMC repeatedly and used an automated pipeline to subtract point-spread-function matched template images from the recent epoch images. The resulting difference images are remarkably clean of the constant (in time) stellar background and are ideal for searching for variable objects. Using these difference images, we have mapped the extensive light echo complex around SN 1987A further out, and deeper, than has been previously possible. Besides the SN 1987A light echoes, we found three other groups of light echoes associated with known supernova remnants (SNRs). These SNRs are three of the six youngest in the LMC, and are classified as likely SN Ia based on X-ray data (Hughes et al. 1995). By combining the position and apparent proper motions of the light echoes, we derive ages for the SNRs (Rest et al. 2005). Spectra of the light echoes taken with the GMOs spectrograph on the Gemini South telescope suggests that the explosion causing one of the SNRs was an overluminous Type Ia supernova explosion.

\section{References}

Crotts, A. 1988, IAUC 4561

Hughes, J. P., Hayashi, I., Helfand, D., et al. 1995, ApJ (Letters), 444, L81

Rest, A., Suntzeff, N. B., Olsen, K., \& SuperMACHO collaboration 2005, Nature, 438, 1132 